\title{
Cardiac Magnetic Resonance and Myocardial Viability: Why Is It so Important?
}

\author{
loana Rodean', Elena Beganu1', Roxana Hodas¹, Elisabeta Himcinschi', Lehel Bordi', \\ Theodora Benedek ${ }^{1,2}$ \\ ${ }^{1}$ Center of Advanced Research in Multimodality Cardiac Imaging, Cardio Med Medical Center, Tîrgu Mureș, Romania \\ 2 University of Medicine and Pharmacy, Tîrgu Mureș, Romania
}

\section{CORRESPONDENCE}

\section{Elena Beganu}

Str. 22 Decembrie 1989 nr. 76

540124 Tîrgu Mureș, Romania

Tel: +40 265217333

E-mail: beganu.elena@yahoo.com

\section{ARTICLE HISTORY}

Received: June 16, 2017

Accepted: July 27, 2017
Ioana Rodean • Str. 22 Decembrie $1989 \mathrm{nr} .76$ 540124 Tîrgu Mureș, Romania. Tel: +40 265217333. E-mail: ioana patricia@yahoo.com

Roxana Hodas • Str. 22 Decembrie 1989 nr. 76 , 540124 Tîrgu Mureș, Romania. Tel: +40 265217333. E-mail: roxana.hodas@yahoo.ro

Elisabeta Himcinschi • Str. 22 Decembrie 1989 nr.76 540124 Tîrgu Mures, Romania. Tel: +40 265217333 E-mail: eli_himcinschi@yahoo.com

Lehel Bordi • Str. 22 Decembrie 1989 nr. 76, 540124 Tîrgu Mures, Romania. Tel: +40 265217 333. E-mail: bordi_lehel:gmail.com

Theodora Benedek • Str. Gheorghe Marinescu nr. 38 540139 Tîrgu Mureș, Romania. Tel: +40 265215551

E-mail: theodora.benedek@gmail.com

\begin{abstract}
For a better assessment of ischemic heart diseases, myocardial viability should be quantified. Current studies underline the importance and the evolution of several techniques and methods used in the evaluation of myocardial viability. Taking into account these considerations, the aim of this manuscript was to present the recent points of view regarding myocardial viability and its clinical significance in patients with ischemic cardiomyopathies and left ventricular dysfunction. On the other hand, the manuscript points out the role of magnetic resonance imaging (MRI), one of the most useful noninvasive imaging techniques, in the assessment of myocardial viability. By comparing the advantages and disadvantages of cardiac MRI, its usefulness can be better appreciated by the clinician. In the following years, it is considered that MRI will be an indispensable imaging tool in the assessment of ischemic heart disease, guiding interventions for revascularization and long-term risk stratification in patients with stable angina or myocardial infarction.
\end{abstract}

Keywords: cardiac magnetic resonance imaging, myocardial viability, myocardial infarction

\section{INTRODUCTION}

Ischemic heart disease remains the main cause of death in adults in western countries despite recent and continuous developments in therapeutic and interventional methods. ${ }^{1,2}$ Although the prevention and treatment of ischemic heart disease have improved significantly, the prevalence of heart failure continues to rise. Ischemic heart diseases present not only an increased prevalence, but also their prognosis is worse compared to non-ischemic cardiomyopathies. ${ }^{3}$ One of the most forceful prognostic factors in patients with coronary artery disease is left ventricular function and its remodeling. Therefore, an early identification of these modifications is necessary in order to guide the treatment and follow-up of these patients. ${ }^{4,5}$ The myocardium is very sensitive to ischemia, thus the degree of contractile damage is influenced by the severity and the duration of the ischemic event, from reversible myocardial tissue to necrosis. Once the myocardium dies, the normal myocardium is replaced by collagenous scar tissue. The 
remodeling process depends on the size and localization of the infarcted area, and it will lead to heart failure. Left ventricular remodeling is defined as a structural, geometri$\mathrm{cal}$, and functional change in the infarct patch and remote viable myocardium. ${ }^{4-6}$ Thus, new treatment options cannot limit ventricular remodeling. It was demonstrated that a coronary intervention improves left ventricular fraction with only $3 \%$ to $4 \%$. $^{7}$

Various factors, such as serum biomarkers (troponin, NT-pro-BNP) as well as angiographic or echocardiographic imaging results have facilitated physicians to estimate the remodeling process. Most of the data can be confirmed by noninvasive imaging modalities, including echocardiography, multi-detector computed tomography (MDCT), magnetic resonance imaging (MRI), positron emission tomography (PET), or single-photon emission computed tomography (SPECT). ${ }^{5,8}$ Recently, cardiac MRI has been recognized as a powerful modality for heart imaging, useful in assessing myocardial structure, function, perfusion, or viability. Cardiac MRI is also an alternative method for risk stratification in patients with ischemic cardiomyopathies (late gadolinium enhancement is correlated with poor prognosis and major cardiovascular events). ${ }^{9-11}$ The assessment of myocardial viability is very important in the management of patients with cardiac ischemic disease. If the percentage of necrotic muscle is not so high, the myocardium can be recovered, thus the prognosis and the functional capacity of the patient can significantly improve following revascularization. ${ }^{12}$

Considering all these assumptions, the purpose of this article is to highlight the importance of myocardial viability and its clinical significance in patients with coronary artery disease and left ventricular dysfunction. Furthermore, the manuscript aims to underline the usefulness of noninvasive imaging techniques in quantifying myocardial viability, especially the role of MRI.

\section{MYOCARDIAL VIABILITY - NEW AND OLD CONCEPTS}

Acute myocardial infarction occurs when a long-drawn reduction of blood flow in a specific heart zone leads to the permanent damage of the myocytes. Myocardial viability has a key role in the risk stratification of patients with ischemic cardiomyopathies, due to its implications on the ventricular remodeling process. The dysfunctional myocardium (viable or non-viable) is one of the most important predictive factors of cardiac function. Thus, the quantification of viable myocardium is of utmost importance in patients with ischemic cardiomyopathies, where the revascularization procedures may improve the left ventricular systolic function and the patient's quality of life. Myocardial viability is defined as a regional wall motion abnormality (hypokinesia, dyskinesia, or akinesia) without the presence of necrotic myocardial tissue. ${ }^{4,6,12}$

After an acute event, the ischemic cascade is triggered, and myocardial viability, especially the reversibility of this process, can be influenced. A series of pathophysiological modifications appear in the damaged myocardium with the intent to replace the dead tissue with fibrotic scar tissue, as the viable myocardium tries to compensate the dysfunctional myocardium in order to maintain a competent cardiac output. Myocardial contractility impairment, followed by intracellular edema, appears within the first few seconds of the acute event. After 30 to 60 minutes from the onset of the event, an irreversible injury of the myocardial cells appears and, if the ischemic process continues, the viable myocardium is replaced by necrotic tissue due to the myocytes' death, followed by a remodeling process that affects left ventricular function. This may lead to progression towards heart failure in years. The progression of myocardial necrosis starts typically from the subendocardium towards the subepicardium (3-6 hours).,13

The myocardium at risk, also named jeopardized myocardium, is one of the principal determinants of the infarcted area, as it forecasts the maximal territory of myocardium exposed to the risk of necrosis. Despite the new reperfusion methods and their beneficial effects, the death of the myocytes can progress during the first hours after reperfusion, through a process named myocardial reperfusion injury. The major cause for this intricate phenomenon is the existence of microvascular obstruction leading to a lack of tissue perfusion despite a successful revascularization procedure. Myocardial territories with normal blood flow in resting conditions may present depressed cardiac function due to sustained ischemic episodes. This phenomenon is called stunning myocardium, and it may be recovered by reperfusion (revascularization or inotropic agents), but it takes days or weeks until it normalizes, once the flow is restored. Viable myocardium with depressed contractility can be partially or completely recovered, due to an impaired coronary blood flow that is called hibernating myocardium. If in the past the hibernating myocardium was thought to appear due to a constant hypoperfusion or mediated by fundamental modifications in myocardial metabolism and energetics (both of them being reduced), nowadays, it is considered that hibernating myocardium is the summation of a continuous and additive stunning, which leads to chronic dysfunction of the left ventricle. Recent studies have shown that hibernating myocardium 
appears due to a shift from fat to glucose metabolism, with the reactivation of the fetal gene program. This type of myocardium contains not only apoptotic cells, but also cells with autophagosomes, vacuoles, and lysozymes. ${ }^{1-4,14}$

\section{ASSESSMENT OF MYOCARDIAL VIABILITY}

In the last years, imaging techniques used to assess and quantify myocardial viability have continuously developed. Nowadays, one of the most significant and useful noninvasive diagnostic methods for assessing the global function of the left ventricle is cardiac MRI, which is able to provide anatomic and ischemia-related imaging with assessment of the consequence of myocardial ischemia, especially on myocardial perfusion, function, and irreversible lesions. ${ }^{15}$ Cardiac MRI also has the ability to evaluate the global and regional left ventricular function following a myocardial infarction, quantifying the infarct size, identifying microvascular obstructions, and evaluating the area at risk. ${ }^{1,16}$

Several MRI techniques can be used for the estimation of myocardial viability. Spectroscopy is used to appraise the cellular metabolites and the integrity of the myocytes. Another technique, able to differentiate between viable and non-viable myocardium, is sodium imaging by MRI. $\mathrm{T} 1$ and $\mathrm{T} 2$ images and maps are useful in evaluating myocardial edema, infarction, or areas at risk. Cinemagnetic resonance highlights segmental and global contractility and wall thickness. Perrone-Filardi et al. have proved that significant parietal thinning may appear also in the viable myocardium. ${ }^{17}$

Myocardial viability can be assessed in three ways: resting MRI - to measure end-diastolic wall thickness; dobutamine stress MRI (DSMR) - to appreciate the contractile reserve; and delayed contrast-enhanced cardiac MRI (DEMRI) - to assess viability. ${ }^{14}$

DSMR identifies the contractile reserve in the hibernating myocardium in response to a certain dose of inotropic agent. At a low dose of dobutamine, the increased contractility is dysfunctional, whereas at higher doses the contractility may decrease, reflecting inducible myocardial ischemia. This "biphasic" response is correlated with post-operative functional recovery. Frequently, scarred segments of the left ventricle do not reveal functional reestablishment in comparison to regions without scars. Dobutamine MRI can estimate viability with enhancement in a dysfunctional wall segment by one grade (from akinetic to hypokinetic or from hypokinetic to normal). Baer et al. showed that an expanded wall thickening greater than $2 \mathrm{~mm}$ during systole is an accurate predictive factor for myocardial recovery. ${ }^{18}$ Another way to quantify viability is dobutamine-induced systolic thickening. Segments with end-diastolic wall thickness less than $5.5 \mathrm{~mm}$ never reveal an improvement of function after revascularization. Even if the wall thickness is bigger than $5.5 \mathrm{~mm}$ after revascularization, an improvement in contractility is not always revealed. ${ }^{3,13,19-21}$

DE-MRI is the most common noninvasive technique that can accurately identify the fibrotic myocardial tissue following a myocardial infarction. Gadolinium-diethylenetriamine pentaacetic acid is the contrast agent used for MRI examinations, which is able to diffuse and accumulate in myocardial tissue. ${ }^{7}$ A multi-center trial assessed the performance of late gadolinium enhancement imaging using a contrast agent (gadolinium) to detect the presence of myocardial infarction. Over 280 patients with myocardial infarction were enrolled in the study, randomized in four groups receiving $0.05,0.10,0.20$, or $0.30 \mathrm{mmol} / \mathrm{kg}$ of gadolinium, respectively. The accuracy of late gadolinium enhancement images regarding the location and quantification of the infarct size and area after a delay of 10-30 minutes was $91 \%$ when administering a dose of $0.20 \mathrm{mmol} /$ $\mathrm{kg}$ and $95 \%$ if the dose was raised to $0.30 \mathrm{mmol} / \mathrm{kg}$. For the $0.10 \mathrm{mmol} / \mathrm{kg}$ dose, the accuracy was $79 \% .22$

This examination is based on the fact that in fibrotic tissues the intercellular space is reduced, and the contrast substance (gadolinium) is accumulated in the extracellular space with a gradient, being excreted over time by the renal system. This way, gadolinium contrast agents can define the transmural extension of the myocardial scar. Due to the different magnetic properties of gadolinium compared to that of blood or myocardial tissue, gadolinium accumulation in the necrotic areas appears enhanced. In acute myocardial infarction, the gadolinium contrast agent is increased in the extracellular space due to cell membrane rupture. Similarly, in old myocardial infarction areas, the contrast agent accumulates in the interstitial space because of the fibrotic tissue that replaces the normal one. ${ }^{23}$ Thus, the infarcted myocardium appears hyperenhanced, the microvascular obstruction zone is hypoenhanced (this phenomenon was observed in $20-50 \%$ of patients after revascularization according to Ahmed et al.), and the normal myocardium appears black (is "nulled"), providing a high contrast between the viable and non-viable myocardial tissue. ${ }^{24,25}$ These modifications are correlated with non-viable myocardium and frequently occur in dysfunctional segments. Due to the possibility to differentiate viable from non-viable tissue, this technique allows the classification of risk associated with revascularization and further complications in patients with ischemic heart disease. ${ }^{2,3,13,19}$ 
As a protocol for DE-MRI, Souto et al. proposed, after obtaining the scout images, to perform a global and segmental functional study of the heart using the cine MRI technique. Ten minutes after the administration of gadolinium, the images of viability could be acquired in the short, long two-chamber, and outflow axes. The acquisitions of delayed enhancement lasts approximately $10 \mathrm{sec}-$ onds and one apnea (the entire examination lasts about 30 minutes). ${ }^{12}$ Usually, the analysis of the myocardium is performed in the short-axis orientation with additional singleslice long axis outlook in 4,3 , and 2 chambers. ${ }^{23}$

Furthermore, to assess and quantify myocardial fibrosis and global viability, MRI evaluates the possible chances of myocardial recovery, attempting to distinguish the 17 segments of the left ventricle. Ortiz-Perez et al. have analyzed the relationship between the 17 segments to identify the late gadolinium enhancement pattern of the culprit lesion on angiography following an acute myocardial infarction. The results of their study revealed a specificity of $100 \%$ for left anterior descending artery occlusion (late gadolinium enhancement was present in the basal anteroseptum, midanterior, anteroseptal, or apical anterior wall). For right coronary artery or left circumflex artery occlusions, no segments were $100 \%$ specific. ${ }^{26}$ Lund et al. demonstrated that delayed enhanced MRI had a higher sensitivity compared to SPECT. ${ }^{27}$

DE-MRI presents a sensitivity of $99 \%$ in measuring the extent of the infarction scar and $94 \%$ in measuring transmural enhancement. The latter can anticipate the recovery of a regional function in an enhanced segment. Myocardial function improves progressively with the decrease of the transmural scar of the infarction. If the myocardium is without any delayed enhancement, the probability of recovery is very high (around $80 \%$ ). Even when the infarcted area is around $1-25 \%$, and the chances of recovery decrease to $60 \%$, viability is still preserved and it remains preserved at a percentage of delayed enhancement of around $26-50 \%$, but a higher percentage is strongly correlated with non-viable myocardial muscle. ${ }^{12,21,28}$

The transmurality of late gadolinium enhancement is an important prognostic factor in an acute myocardial infarction. Bodi et al. demonstrated that there is a meaningful difference between major cardiovascular events when comparing patients with 5 or more segments of transmural infarction with those with less involved myocardium (23\% vs. $5 \%, \mathrm{p}<0.001) .{ }^{29}$ Furthermore, Wu et al. have shown that left ventricular remodeling is a more powerful predictor of clinical events than systolic function in patients with acute myocardial infarction. During the 4-month MRI follow-up, all patients enrolled in this study presented an im- provement in the ejection fraction $(\mathrm{p}=0.002)$. One of the most important conclusions of this study was that acute infarct size is correlated directly with the left ventricular remodeling process. ${ }^{30}$ Buckert et al. have confirmed that the presence of late gadolinium enhancement is a strong clinical predictor of cardiovascular events in patients with stable coronary artery disease..$^{10,31,32}$

Rizzi et al. have demonstrated in the MESA study that several techniques can be used to quantify infarction dimension, such as: visual technique, planimetry, the standard deviation technique, full width at half maximum, and the correction of image noise. ${ }^{33}$ Semiautomatic analysis with manual correction and removal of artifacts is the first choice among diagnostic methods to quantify the infarction zone and fibrosis. ${ }^{12}$

The size of myocardial infarction in the acute phase differs from the one in the chronic phase due to the fact that in acute infarction the affected myocardial mass is up to $25 \%$ bigger as a result of tissue edema, hemorrhage, and inflammation. ${ }^{34}$ According to Ingkanisorn et al., chronic infarct size is significantly smaller 2 months after the acute infarction ( $p<0.003)$. Furthermore, in a study carried out by Choi et al., after a period of 8 weeks from the acute event, the size of infarction decreased with $26 \%$. A greater degree of infarct involution was present in infarcts with a higher degree of microvascular obstruction, possibly due to a more severe initial ischemic lesion..$^{35}$

Microvascular obstructions should be quantified due to their association with the "no-reflow" phenomenon. This microvascular modification occurs only in acute infarction and persists for at least 9 days. ${ }^{36}$ Its existence can be identified using MRI techniques, appearing as a default of subendocardial contrast uptake in an area surrounded by hyperenhancement, or as early hypoenhancement observed within the first few minutes after first-pass contrast perfusion (early enhancement imaging). ${ }^{37}$ As Rogers et al. have demonstrated in their study, microvascular obstructions are predictors of non-viable myocardium, because in the regions with microvascular obstruction there was no recovery of segmental left ventricular function. ${ }^{38}$ In addition, the presence of microvascular obstructions was correlated with a higher incidence of cardiovascular events and was strongly associated with left ventricular remodeling. ${ }^{16}$ In this regard, Hombach et al. found that microvascular obstruction is an important predictor of left ventricular remodeling, ejection fraction, and survival following an acute myocardial infarction. ${ }^{39}$

Recent studies proved that dobutamine-stress MRI better forecasts functional recovery than late gadolinium enhancement. In 2005, Tarantini et al. have published one 
of the first studies that associated the time of coronary lesion, the degree of microvascular obstruction, and transmural necrosis. In this research, the patients were stratified based on TIMI grade flow obtained after myocardial revascularization. Regarding the related lag in reperfusion for both groups (group with TIMI 3 grade flow and group with TIMI grade flow <3), there was a greater number of patients with microvascular obstruction and transmural necrosis in the latter group. For every 30-minute delay in a successful percutaneous coronary intervention, a continuum of microvascular obstruction and myocardial necrosis was observed on MRI in the group with a TIMI grade flow $<3$, which was correlated with a $37 \%$ higher risk of transmural necrosis and a $12 \%$ higher risk of microvascular obstruction. ${ }^{40} \mathrm{Wu}$ et al. argued that the presence of microvascular obstruction (residual myocardial perfusion abnormality despite revascularization of the tissue) is an important marker for injury severity. ${ }^{41}$ Furthermore, Roses et al. established that the expansion of the microvascular obstruction zone is a better long-term prognostic factor compared to the ejection fraction and left ventricular volumes. ${ }^{42}$

The MRI examination can determine the area at risk and the infarct size. T2-weighted MRI aids visualization of the risk area, which appears hyperintense compared to the normal myocardium. The $\mathrm{T} 2$ sequence is also useful in differentiating between acute and chronic infarction. ${ }^{20,43}$ The quantification of areas at risk is very important, because they are markers for reversible injury. Another MRI sequence that can be used to appreciate the size of the myocardial area at risk and to differentiate between acute and chronic myocardial infarction is T2-weighted short-tau inversion recovery (STIR). ${ }^{44}$ Abdel-Aty et al. have compared patients with chronic and acute myocardial infarction, and the results have shown that patients with chronic infarction do not present any signal-to-noise ratio between the infarcted and the healthy myocardium on T2-weighted imaging. ${ }^{45}$ There are several studies that showed that $\mathrm{T} 1-$ mapping is useful in identifying more diffuse forms of fibrosis in the remote myocardium and the peri-infarct area (the acquisition time is faster and the spatial resolution is better). ${ }^{46,47}$

The notion of peri-infarct zone ("gray zone") was introduced by Schmidt $e t$ al. The quantification of the peri-infarcted zone can be made by tracing the endo- and epicardial margins in the short-axis sequence and the hypersignal region. ${ }^{41}$ The infarct nucleus is defined as the region from the myocardium with a signal intensity higher than $50 \%$. The gray zone is described as a zone with greater signal intensity than that of the remote myocardium but lower than $50 \%$. This zone refers to the region of intermediate late gadolinium enhancement from the myocardial scar periphery, which is related to the possibility of inducing arrhythmias. Additionally, Roes et al. established that the presence of peri-infarct tissue heterogeneity in patients with myocardial infarction is a pronounced predictive factor for ventricular arrhythmia and ICD therapy compared to the total infarct size and left ventricular function. ${ }^{16}$

Several trials on myocardial viability in patients with coronary artery disease have been performed, but their results remain controversial. The recent STICH trial (Surgical Treatment for Ischemic Heart Failure) does not show the advantage of revascularization compared to medical therapy in patients with ischemic cardiomyopathies. Despite its limitations, the STICH trial remains one of the largest analyses of the influence of viability on clinical endpoints in subjects with ischemic heart diseases. Another large trial that was of use in the evaluation of myocardial viability, risk stratification, and in the identification of patients who could benefit from revascularization, is the PAAR-2 (PET and Recovery Following Revascularization) trial, but this study failed to prove a difference in primary outcomes at one year. Ottawa-FIVE (18F-FDG PET Imaging of Myocardial Viability in an Experienced Center with Access to 18F-FDG and Integration with Clinical Management Teams) is a PARR-2 sub-study that proved a significant advantage in outcomes in the PET-guided group. The results of this sub-study revealed the prognostic importance of the FDG PET viability imaging technique in patients with ischemic heart disease. ${ }^{4,23}$

\section{ADVANTAGES AND DISADVANTAGES OF CARDIAC MRI}

Despite the fact that many noninvasive imaging modalities have been introduced in the last years for the quantification of myocardial viability, cardiac MRI remains the gold standard imaging technique for diagnosing ischemic heart disease.

The main advantage of cardiac MRI is the fact that it does not present acoustic window limitations. It offers a higher spatial and temporal resolution than other nuclear methods and also a better tissue characterization. This technique is ionizing radiation-free, which is another important advantage compared to computed tomography or nuclear techniques. ${ }^{2}$

Related to echocardiography, cine MRI sequence enables dynamic images of wall motion, with a superior endocardial border assessment. In clinical practice or as an endpoint in clinical trials, MRI can quantify left ventricular 
function and ventricular mass (it can be measured in cine MRI sequence). There are studies that have demonstrated a higher accuracy of high-dose dobutamine MRI compared to high-dose dobutamine stress echocardiography ( $86 \%$ vs. $72 \%) .49,50$

Using perfusion MRI, myocardium viability and critical coronary lesions can be detected and several perfusion indices can be calculated (curve upslope, maximum signal intensity, and time to peak). Delayed-enhancement MRI is one of the finest techniques used to identify myocardial viability and to quantify the infarcted area. Furthermore, in the T2-weighted sequence, the area of risk or microvascular obstructions can be appreciated. Cardiac MRI examination can be a useful tool in patient follow-up before or after revascularization interventions. ${ }^{2,51}$

For an optimal image acquisition, several rules are necessary. It is important to have a proper temporal resolution to image a few short axis slices for each heartbeat, in order to visualize the initial contrast bolus. High spatial resolution is another important factor that must be taken into consideration due to the necessity to discern between the subendocardial and subepicardial myocardium. ${ }^{52}$

The main disadvantages of this technique include the MRI breath-holds sequence, time examination, contrast agent, adverse reactions, and costs. Renal disease represents another limitation of MRI, due to the risk of systemic nephrogenic fibrosis (if eGFR $<30 \mathrm{~mL} / \mathrm{min} / 1.73 \mathrm{~m}^{2}$ ). ${ }^{2,3}$

Persons with electrically, magnetically, or mechanically activated devices and metallic or ferromagnetic implants (pacemakers, insulin pumps, defibrillators, cochlear implants etc.) cannot undergo MRI examinations. Recent studies have demonstrated that newer stents or cardiac devices are compatible with MRI, but their safety must be checked by the physician. For a good MRI examination, an optimal patient cooperation is necessary, thus claustrophobia, obesity, or unstable patients may limit the examination. ${ }^{51}$

\section{CONCLUSION}

Ischemic heart disease remains an important health problem due to its high mortality. The healing process after an acute myocardial infarction is a dynamic one that can influence heart function, left ventricular remodeling, and disease progression. Myocardial viability is the key point in revascularization techniques, guiding the treatment and patient prognosis; therefore, it should be quantified in each patient with myocardial ischemia.

Cardiac MRI represents the gold standard noninvasive diagnostic method in assessing myocardial viability and in the long-term follow-up of patients with ischemic cardiac disease, especially in the risk stratification of patients with myocardial infarction. Both in the acute and chronic phase, DE-MRI can quantify the size of the infarcted area and is able to estimate whether there is viable myocardium in that region. This examination can also identify microvascular obstructions or the area at risk, it can characterize the infarcted area and myocardial necrosis markers, and it may forecast the risk of death in patients with ischemic heart disease. Also, MRI provides complementary information about global and regional cardiac function and perfusion.

In the future, MRI will be an indispensable imaging tool for the quantification of ischemic heart disease, for guiding the revascularization intervention, and for longterm risk stratification in patients with chest pain, stable angina, or myocardial infarction, due to its ability to provide high definition images of anatomical and functional heart characteristics in real time, without exposure to ionizing radiation.

\section{CONFLICT OF INTEREST}

Nothing to declare.

\section{REFERENCES}

1. Florian A, Jurcut R, Ginghina C, Bogaret J. Cardiac Magnetic Resonance Imaging in Ischemic Heart Disease - A Clinical Review. J Med Life. 2011;4:330-345

2. Saeed M, Van TA, Krug R, Hetts SW, Wilson MW. Cardiac MR imaging: current status and future direction. Cardiovasc Diagn Ther. 2015;5:290310 .

3. Partington SL, Kwong RY, Dorbala S. Multimodality imaging in the assessment of myocardial viability. Heart Fail Rev. 2011;16:381-395.

4. Bhat A, Gan GC, Tan TC, Hsu C, Denniss AR. Myocardial Viability: From Proof of Concept to Clinical Practice. Cardiol Res Pract. 2016;2016:1020818.

5. Kim EK, Song YB, Chang SA, et al. Is cardiac magnetic resonance necessary for prediction of left ventricular remodeling in patients with reperfused ST-segment elevation myocardial infarction? Int J Cardiovasc Imaging. 2017. doi: 10.1007/s10554-017-1206-z. [Epub ahead of print]

6. Richardson WJ, Clarke SA, Quinn TA, Holmes JW. Physiological Implications of Myocardial Scar Structure. Compr Physiol. 2016;5:1877-1909.

7. Mester A, Oltean-Péter B, Rodean I, et al. Magnetic Resonance Imaging of Myocardial Function Following Intracoronary and Intramyocardial Stem Cell Injection. Journal of Interdisciplinary Medicine. 2017;2:112-116.

8. Bax JJ, Delgado V. Myocardial viability as integral part of the diagnostic and therapeutic approach to ischemic heart failure. J Nucl Cardiol. 2015;22:229-245

9. Saeed M, Liu H, Liang $\mathrm{CH}$, Wilson MW. Magnetic resonance imaging for characterizing myocardial diseases. Int J Cardiovasc Imaging. 2017. doi: 10.1007/s10554-017-1127-x. [Epub ahead of print]

10. Buckert D, Kelle S, Buss S, et al. Left Ventricular Ejection Fraction and Presence of Myocardial Necrosis Assessed by Cardiac Magnetic Resonance Imaging Correctly Risk Stratify Patients With Stable Coronary Artery Disease: A Multi-Center All-Comers Trial. Clin Res Cardiol. 2016;106:219-229.

11. Hassanein AS, Khalifa AM, Ibrahim el-SH. Cine Viability Magnetic Resonance Imaging of the Heart Without Increased Scan Time. Magn Reson Imaging. 2015;34:183-190

12. Souto ALM, Souto RM, Teixeira ICR, Nacif MS. Myocardial Viability on Cardiac Magnetic Resonance. Arq Bras Cardiol. 2017;108:458-469. 
13. Masci PG, Bogaert J. Post myocardial infarction of the left ventricle: the course ahead seen by cardiac MRI. Cardiovasc Diagn Ther. 2012;2:113-127.

14. Shabana A, El-Menyar A. Myocardial Viability: What We Knew and What Is New. Cardiol Res Pract. 2012;2012:607486.

15. Bordi L, Kovacs I, Bajka B, et al. New Imaging-based Tools for the Assessment of Ventricular Function in Ischemic Heart Diseases. Journal of Interdisciplinary Medicine. 2017;2:31-35.

16. West AM, Kramer CM. Cardiovascular Magnetic Resonance Imaging of Myocardial Infarction, Viability, and Cardiomyopathies. Curr Prob/ Cardiol. 2010;35:176-220

17. Perrone-Filardi P, Bacharach SL, Dilsizian V, et al. Metabolic evidence of viable myocardium in regions with reduced wall thickness and absent wall thickening in patients with chronic ischemic left ventricular dysfunction. $J$ Am Coll Cardiol. 1992;20:161-168.

18. Bove CM, DiMaria JM, Voros S, Conaway MR, Kramer CM. Dobutamine response and myocardial infarct transmurality: functional improvement after coronary artery bypass grafting-initial experience. Radiology. 2006:240:835-841.

19. Health Quality Ontario. Magnetic resonance imaging (MRI) for the assessment of myocardial viability: an evidence-based analysis. Ont Health Technol Assess Ser. 2010;10:1-45

20. Arai AE. The cardiac magnetic resonance (CMR) approach to assessing myocardial viability. J Nucl Cardiol. 2011;18:1095-1102

21. Schinkel AF, Poldermans D, Elhendy A, Bax JJ. Assessment of myocardial viability in patients with heart failure. J Nucl Med. 2007;48:1135-1146.

22. Kim RJ, Albert TSE, Wible JH, et al. Performance of delayed-enhancement myocardial resonance imaging with gadoversetamide contrast for the detection and assessment of myocardial infarction. Circulation. 2008;117:629-637.

23. Jimenez JL, Crean AM, Wintersperger BJ. Late gadolinium enhancement imaging in assessment of myocardial viability: techniques and clinical applications. Radiol Clin North Am. 2015:53:397-411.

24. Ahmed N, Carrick D,Layland J, Oldroyd KG, Berry C. The role of cardiac magnetic resonance imaging (MRI) in acute myocardial infarction (AMI). Heart Lung Circ. 2013;22:243-255.

25. Rubenstein JC, Lee DC, Wu E, et al.A comparison of cardiac magnetic resonance imaging peri-infarct border zone quantification strategies for the prediction of ventricular tachyarrhythmia inducibility. Cardiol $\mathrm{J}$. 2013;20:68-77.

26. Ortiz-Perez JT, Rodriguez J, Meyers SN, Lee DC, Davidson C, Wu E. Correspondence between the 17-segment model and coronary arterial imaging using contrast-enhanced cardiac magnetic resonance imaging. $J$ Am Coll Cardiol Img. 2008;1:282-293.

27. Lund GK, Stork A, Saeed M, et al. Acute myocardial infarction: evaluation with first-pass enhancement and delayed enhancement MR imaging compared with 201TI SPECT imaging. Radiology. 2004;232:49-57.

28. Saeed M, Hetts SW, Jablonowski R, Wilson MW. Magnetic resonance imaging and multi-detector computed tomography assessment of extracellular compartment in ischemic and non-ischemic myocardial pathologies. World J Cardiol. 2014;6:1192-1208

29. Bodi V, Sanchis J, Nunez J, et al. Prognostic value of a comprehensive cardiac magnetic resonance assessment soon after a first ST-segment elevation myocardial infarction. J Am Coll Cardiol Img. 2009;2:835-842.

30. Wu E, Ortiz JT, Tejedor $P$, et al. Infarct size by contrast enhanced cardiac magnetic resonance is a stronger predictor of outcomes than left ventricular ejection fraction or end-systolic volume index: prospective cohort study. Heart. 2008;94:730-736.

31. Buckert D, Dewes P, Walcher T, et al. Intermediate-term prognostic value of reversible perfusion deficit diagnosed by adenosine CMR: a prospective follow-up study in a consecutive patient population. JACC Cardiovasc Imaging. 2013;6:56-63.

32. Zemrak F, Petersen SE. Late gadolinium enhancement CMR predicts adverse cardiovascular outcomes and mortality in patients with coronary artery disease: systematic review and meta-analysis. Prog Cardiovasc Dis. 2011:54:215-229.

33. Rizzi PB, Nacif M, Volpe GJ, et al. Quantification of myocardial scar assessed by late gadolinium enhancement CMR in the multi-ethnics study of atherosclerosis: comparisons of 7 different methods. J Cardiovasc Magn Reson. 2013;15:049
34. Reimer KA, Jennings RB. The changing anatomic reference base of evolving myocardial infarction.Underestimation of myocardial collatera blood flow and overestimation of experimental anatomic infarct size due to tissue edema, hemorrhage and acute inflammation. Circulation. 1979;60:866-876

35. Choi CJ, Haji-Momenian S, DiMaria JM, et al. Infarct involution and improved function during healing of acute myocardial infarction: the role of microvascular obstruction. J Cardiovasc Magn Reson. 2004;6:917-925.

36. Wu KC, Kim RJ, Bleumke DA, et al. Quantification and time course of microvascular obstruction by contrast-enhanced echocardiography and magnetic resonance imaging following acute myocardial infarction and reperfusion. J Am Coll Cardiol. 1998;32:1756-1764.

37. Lima JAC, Judd RM, Bazille A, et al. Regional Heterogeneity of Human Myocardial Infarcts Demonstrated by Contrast-Enhanced MRI. Circulation. 1995;92:1117-1125

38. Rogers WJ Jr, Kramer CM, Geskin G, et al. Early contrast-enhanced MRI predicts late functional recovery after reperfused myocardial infarction. Circulation. 1999;99:744-750.

39. Kramer CM. The prognostic significance of microvascular obstruction after myocardial infarction as defined by cardiac magnetic resonance. Eur Heart J. 2005;26:532-533.

40. Tarantini G, Cacciavillani L, Corbetti F, et al. Duration of ischemia is a major determinant of transmurality and severe microvascular obstruction after primary angioplasty: a study performed with contrast-enhanced magnetic resonance. J Am Coll Cardiol. 2005;46:1229-1235.

41. Deneke T, Muller KM, Lemke B, et al. Human histopathology of electroanatomic mapping after cooled-tip radiofrequency ablation to treat ventricular tachycardia in remote myocardial infarction. J Cardiovasc Electrophysiol. 2005;16:1246-1251.

42. Roes SD, Kelle S, Kaandorp TA, et al. Comparison of myocardial infarct size assessed with contrast-enhanced magnetic resonance imaging and left ventricular function and volumes to predict mortality in patients with healed myocardial infarction. Am J Cardiol. 2007;100:930-936.

43. Wu KC, Zerhouni EA, Judd RM, et al. Prognostic significance of microvascular obstruction by magnetic resonance imaging in patients with acute myocardial infarction. Circulation. 1998:97:765-772.

44. Friedrich MG, Abdel-Aty $\mathrm{H}$, Taylor A, et al. The salvaged area at risk in reperfused acute myocardial infarction as visualized by cardiovascular magnetic resonance. J Am Coll Cardiol. 2008;51:1581-1587.

45. Abdel-Aty H, Zagrosek A, Schulz-Menger J, et al. Delayed enhancement and T2-weighted cardiovascular magnetic resonance imaging differentiate acute from chronic myocardial infarction. Circulation. 2004;109:2411-2416.

46. Dall'Armellina E, Piechnik SK, Ferreira VM, et al. Cardiovascular magnetic resonance by non- contrast T1-mapping allows assessment of severity of injury in acute myocardial infarction. J Cardiovasc Magn Reson. 2012;14:15.

47. Ugander M, Oki AJ, Hsu LY, et al. Extracellular volume imaging by magnetic resonance imaging provides insights into overt and subclinical myocardial pathology. Eur Heart J. 2012;33:1268-1278.

48. West AM, Kramer CM. Cardiovascular Magnetic Resonance Imaging of Myocardial Infarction, Viability, and Cardiomyopathies. Curr Prob/ Cardiol. 2010;35:176-220

49. Nagel E, Lehmkuhl HB, Bocksch W, et al. Noninvasive diagnosis of ischemia-induced wall motion abnormalities with the use of high dose dobutamine stress MRI: comparison with dobutamine stress echocardiography. Circulation. 1999;99:763-770.

50. Hundley WG, Hamilton CA, Clarke GD, et al. Visualization and functional assessment of proximal and middle left anterior descending coronary stenoses in humans with magnetic resonance imaging. Circulation. 1999;99:3248-3254.

51. Morariu M, Bordi L, Opincariu D, Ratiu M, Condrea S, Benedek A, Benedek T. New Developments in Magnetic Resonance Imaging of Myocardial Diseases - Technical Aspects and Clinical Applications. Journal of Interdisciplinary Medicine. 2017;2:17-21.

52. Barkhausen J, Hunold P, Jochims M, Debatin JF. Imaging of myocardial perfusion with magnetic resonance. J Magn Reson Imaging. 2004;19:750757. 